\title{
A systematic review of theoretical constructs in CDS literature
}

\author{
Siru Liu*, Thomas J. Reese, Kensaku Kawamoto, Guilherme Del Fiol and Charlene Weir
}

\begin{abstract}
Background: Studies that examine the adoption of clinical decision support (CDS) by healthcare providers have generally lacked a theoretical underpinning. The Unified Theory of Acceptance and Use of Technology (UTAUT) model may provide such a theory-based explanation; however, it is unknown if the model can be applied to the CDS literature.

Objective: Our overall goal was to develop a taxonomy based on UTAUT constructs that could reliably characterize CDS interventions.

Methods: We used a two-step process: (1) identified randomized controlled trials meeting comparative effectiveness criteria, e.g., evaluating the impact of CDS interventions with and without specific features or implementation strategies; (2) iteratively developed and validated a taxonomy for characterizing differential CDS features or implementation strategies using three raters.

Results: Twenty-five studies with 48 comparison arms were identified. We applied three constructs from the UTAUT model and added motivational control to characterize CDS interventions. Inter-rater reliability was as follows for model constructs: performance expectancy $(k=0.79)$, effort expectancy $(k=0.85)$, social influence $(k=0.71)$, and motivational control $(\mathrm{k}=0.87)$.

Conclusion: We found that constructs from the UTAUT model and motivational control can reliably characterize features and associated implementation strategies. Our next step is to examine the quantitative relationships between constructs and CDS adoption.
\end{abstract}

Keywords: Clinical decision support, Unified Theory of Acceptance and Use of Technology, Taxonomy

\section{Background}

Clinical decision support (CDS) implemented in the context of an electronic health record (EHR) can enhance patient health and healthcare quality $[1,2]$. CDS has been defined as the provision of patient-specific knowledge, information, and recommendations to clinicians in order to support optimal healthcare decisions [3]. Unfortunately, of published CDS interventions, approximately half are not associated with statistically significant improvements to clinical outcomes [4]. One possible

*Correspondence: siruliu@utah.edu

Department of Biomedical Informatics, University of Utah, Salt Lake City, Utah, USA reason for this variation in outcomes is user adoption. To understand the features that impact adoption, and, in turn, successful patient outcomes, we need more research to investigate the psychological mechanisms that are key to these types of interventions.

Previous systematic reviews have identified features that might increase CDS adoption [5-9]. For example, some CDS features found to have a positive impact include automatic data gathering and presentation, triggering presentation during decision making, and the provision of actionable recommendations [5]. While these systematic reviews do provide insight regarding particular features that may help CDS adoption, they don't directly address the theoretical mechanisms of action. As 
a result, generalizable knowledge is limited. Specifically, reviews on existing features lack theoretical grounding in terms of identifying the relationship between how interventions are operationalized and the causal mechanisms that are being manipulated. This issue has been discussed for many years. As Shekelle et al. stated in their RAND's comprehensive review of health information technology (HIT) interventions:

\section{"In summary, we identified no study or collection of studies, outside of those from a handful of HIT leaders, that would allow a reader to make a deter- mination about the generalizable knowledge of the system's reported benefit. This limitation in gener- alizable knowledge is not simply a matter of study design and internal validity. Even if further rand- omized, controlled trials are performed, the gener- alizability of the evidence would remain low unless additional systematic, comprehensive, and relevant descriptions and measurements are made regarding how the technology is utilized, the individuals using it, and the environment it is used in [8]."}

The pathway from the CDS intervention to adoption behavior begins with the psychological mechanism that is the core functionality of the system, through user beliefs, goals, intentions, and finally behavior. The Unified Theory of Acceptance and Use of Technology (UTAUT) model is a validated integrative model of theories, which can explain more than $70 \%$ of the variance in behavioral intention and $50 \%$ of the variance in user behavior $(\mathrm{N}=399)$ [9]. The UTAUT model was developed by mapping and integrating 32 constructs over 8 well-known adoption theories. Intentions were used because a longstanding body of research has demonstrated that intentions were highly correlated with actual behavior [9]. The UTAUT model has been well validated across many systems. In healthcare, several studies have used the UTAUT model to explain and predict the IT acceptance, such as EHRs and online reporting systems. This body of work provides a reasonable and solid basis for applying the model to the CDS context [10-12].

We propose to use the UTAUT constructs to explore the psychological characteristics of CDS interventions and to predict adoption. The literature on CDS contains very few direct tests or manipulations of specific attributes that directly test a psychological mechanism. For example, CDS content is assumed to be better adopted if the information provided is relevant. However, few studies directly compare low versus high relevant information to test that hypothesis. Our goal is to explore the feasibility of using the UTAUT model to estimate the underlying mechanisms that might be manipulated in a head-to-head comparison of two forms of a CDS intervention. Although the UTAUT constructs were not directly manipulated, we propose to estimate them from the descriptions of the intervention itself, not through individual subjective assessments. In other words, we characterized the manipulation itself using the UAUT taxonomy.

We sought to adapt the UTAUT model to actual features that were tested in randomized controlled trial (RCT) studies by comparing one form of the CDS with another form and categorizing the difference between two groups; we only used RCTs, so the effect found can be rigorously assumed to be due to the manipulated factor. Instead of comparing the usual care with CDS interventions, comparative effectiveness studies use at least two different forms of active interventions (not usual care) to identify what intervention works best to improve outcomes. This restriction on the study design could help us to get the most accurate evidence [11] and use actual usage to measure adoption.

The objectives for this work are to examine the theoretical mechanisms of features influencing providers' CDS adoption and to develop a taxonomy to describe interventions based on the current status of head-tohead comparative randomized evidence. To illustrate usage of the taxonomy, we provide three cases where CDS features are described and coded. This study is expected to contribute in both the technology adoption and CDS domains.

\section{Methods}

This measurement study largely focuses on a conceptual approach, proposed by Bailey, for developing taxonomies [13]. We used constructs grounded in theory from the UTAUT model and the CDS literature.

\section{UTAUT}

The UTAUT model includes four core constructs: (1) performance expectancy; (2) effort expectancy; (3) social influence; and (4) facilitating conditions [13]. Some of these are easily identifiable in the method sections of research studies and some are less so.

Performance expectancy refers to the user's belief that the CDS intervention can improve work performance. It involves five sub-constructs: perceived usefulness, extrinsic motivation (reward), job-fit, relative advantage, and beliefs about expected outcome. Based on the UTAUT model, performance expectancy is the strongest predictor of user intentions [9]. CDS tools are designed to be useful, but few studies actually measure performance expectancy, nor do they directly compare systems with different levels of performance expectancy. In the context of CDS interventions, level of performance expectancy can be determined by using direct evidence from 
interviews, usability surveys, or evidence of user-centered design processes.

Effort expectancy refers to the perceived ease of use, or perceived effort using the CDS intervention. Effort expectancy integrates constructs that include perceived ease of use, complexity, self-efficacy, and anxiety [9]. Effort expectancy can be estimated by the time burden or need for sustained attention. A CDS intervention that decreases the number of clicks or provides more information in one place would be expected to have lower effort. Effort expectancy significantly affects early behavioral intentions, and the effect fades over time as users gain skills in using the CDS [9].

Social influence refers to the degree of social pressure or social expectations to use the CDS intervention. Social influence consists of two parts, leadership pressures and peer social influence [9]. Social influence is also related to subjective norms (internalized beliefs of others' expectations), and perceived image of oneself by others. The level of social influence was determined by several factors, including tools to monitor usage, the provision of performance feedback, comparison of user's behavior with others, and programs to monitor compliance.

Facilitating conditions encompass the infrastructure (both technical and organizational) and the strategies used to support and implement the CDS intervention. Instead of affecting behavioral intentions (i.e., performance expectancy, effort expectancy, and social influence), facilitating conditions can predict actual usage directly [9]. For CDS interventions, we considered facilitating conditions to be estimated in four parts: (1) IT infrastructure or user customization, such as the use of tailoring text or highlighting important information; (2) support for users, such as the provision of technical assistance to address hardware and software issues; (3) training, such as offering educational programs regarding how to use the tool; and (4) other facilitating factors, such as incentives to decrease the cost of unnecessary tests.

Motivational control is a construct that we included to account for human agency, a factor that many researchers believe to be understudied in the area of clinical IT interventions. In addition, clinicians often complain that current EHR systems limit their ability to choose and act with agency [14]. This construct is similar to the construct of autonomy, a core and ubiquitous drive of humans as identified in Self-Determination Theory [15], Bandura's Social Cognitive Theory [16] as well as Csikszentmihalyi's Intrinsic Motivational Theory [17]. Because autonomy is a basic characteristic of professional roles, it is highly relevant to a discussion of the features associated with CDS adoption and use by clinicians. Perceived threat to professional autonomy has been identified as a critical negative impact on physicians' acceptance of healthcare IT [18-22]. Perceived control has a long history in motivational psychology. For example, it was added to the Theory of Planned Behavior [23] to improve the predictive power of the Theory of Reasoned Action [24]. Validation studies of UTAUT addressed control construct by separating the validation into those studies where users could choose to the system versus those studies where users could not choose.

\section{Data sources}

We used an iterative process to develop the taxonomy. First, we selected RCTs from multiple literature reviews related to CDS adoption features published after 2000 [6, 25-27]. We included only RCTs with a direct comparison of features that may affect CDS adoption. This restriction on the study design would improve the accuracy of the taxonomy by using the highest level of evidence [11]. The completed inclusion criteria were: (1) RCTs; (2) comparison of two forms of a CDS tool (the CDS tool with and without additional features); (3) providers received the CDS intervention; (4) examined CDS is integrated into the EHR; (5) all types of CDS: alerts, reminders, order sets, dashboards, infobuttons, documentation templates, and shared decision making tools [3]; (6) automatic extraction and use of data from the EHR; and (7) CDS that was used in inpatient acute care hospitals and in outpatient primary and sub-specialty care clinics. Two authors (SL and TR) participated in study selection and a third author $(\mathrm{CW})$ resolved discrepancies. We extracted study characteristics (e.g., study design, setting, country, clinical area, and CDS type), detailed information including screenshots about each CDS intervention, and any information relating to the constructs.

\section{Taxonomy development}

The preliminary taxonomy was based on the description of the UTAUT model $[9,23,28]$. The coding protocol began initially with the UTAUT model, and followed with an inductive review within each construct through group-based discussion. The iterative process started with two reviewers (SL and TR) independently coding 2-3 studies. Each study arm was coded with constructs in the UTAUT model and motivational control. CDS features were mapped to three levels of intensity (i.e., High, Medium, and Low) within constructs except for facilitating conditions. In developing a measurement protocol for the construct of "facilitating conditions," we found that they were usually listed as parallel implementation strategies, making it difficult to determine which strategy facilitated and certainly we did not usually have any measures of support. We also did not have any direct measures of perceived support. Based on this concern, we counted how many implementation strategies used in the study as 
the coding measurement protocol, but fully understanding this limitation. Hopefully in the future, researchers will do a more thorough job of reporting and testing different strategies. Facilitation is key to implementation based on guidelines developed by the Agency for Healthcare Policy and Research (AHCPR) and is a core component in several implementation theories such as Consolidated Framework for Implementation Research (CFIR) [29], Promoting Action on Research Implementation in Health Services (PHARIS) [30], etc. In addition, studies that examine user's perception of support and the relationship of those perceptions to actual strategies have shown significant relationship. As a result, counting facilitating conditions is a reasonable proxy. Additionally, we compared the CDS intervention coding between study arms. The group (SL, TR, and CW) met and discussed coding discrepancies and revised the taxonomy with each set of 2-3 studies. This process was repeated until two sets of subsequent studies did not result in taxonomy changes and achieved high inter-rater reliability (Cohen's $\mathrm{K}>0.80)$ [31]. An expert in social psychology theory $(\mathrm{CW})$ discussed and adjudicated differences to help ensure the coding quality. To validate the taxonomy, a new set of studies was independently coded by two authors (SL and TR).

\section{Results}

We identified 25 studies meeting the inclusion criteria. Within the included studies, 48 comparison arms were coded. Most studies used a cluster RCT design (88\%). The mean number of sites, number of subjects, and study time (months) were $15.2,16,150$, and 14.7 , respectively. Studies were conducted in the United States (18), the Netherlands (3), Canada (2), New Zealand (1), and the United Kingdom (1). Twenty-three studies (92\%) were conducted in an outpatient setting and two in an inpatient setting. Practice settings included general practice, gynecology, and pediatrics. Table 1 provides characteristics of the CDS interventions.

\section{Taxonomy results}

After four iterations and 10 studies, we reached an acceptable agreement for each construct. The taxonomy was validated by coding an additional 15 studies. Table 2 provides the taxonomy validation agreement and the coding rules for each level within constructs. The coding schema had two parts for each construct: (1) the operational definition of the construct in terms of CDS context and (2) rules to assess levels of intensity using an ordinal scale in order to determine the degree of performance expectancy, effort expectancy, social influence, or motivational control, as well as to extract facilitating conditions. The average inter-rater reliability was $\kappa=0.81$,
Table 1 Characteristics of included CDS interventions

\begin{tabular}{lc}
\hline Characteristic & Percentage (\%) \\
\hline Clinical domain $(n=25)$ & \\
Management of chronic medical condition or preven- & 48 \\
$\quad$ tive care & 36 \\
Pharmacotherapy & 8 \\
Laboratory test ordering & 4 \\
Diagnosis & 4 \\
Immunization & 4 \\
Management of psychiatric condition & \\
Clinical decision support type $(n=25)$ & 60 \\
Alert & 24 \\
Reminder & 16 \\
Order set & 16 \\
Dashboard & 4 \\
Infobutton &
\end{tabular}

which shows high levels of agreement across constructs [32].

\section{Case examples}

We selected three cases to illustrate how the taxonomy and coding schema (Table 2) were used to characterize the CDS interventions. We chose these three cases to illustrate the breath of constructs impacted. The first case illustrates the nature of facilitating conditions; the second case illustrates effort expectancy and motivational control; and the last case illustrates how all of the constructs except performance expectancy can be affected. These examples demonstrate how additional features can interact together, and how changing one construct level may cause other constructs to change.

The first case aimed to test the effect of adding more contextual information into a CDS tool in the outpatient setting [36] (Table 3). The control arm was a drug-drug interaction alert related to hyperkalemia. The intervention was the same alert but with added specific patient laboratory data. Applying our model to compare these two arms, we found that the additional feature, presenting lab data, adds only one facilitating condition and the other four constructs were unchanged. In both arms, performance expectancy was low because researchers did not report usability testing or interviews. In addition, clinicians in the discussion group determined that provided information was not helpful for users. The drug-drug interaction alert was quick and required less than one second to use. Therefore, effort expectancy was low, and motivational control was medium in both arms because users had to respond to the alert. No education program or compliance tracking system was provided, leading to low social influence in this experiment. 
Table 2 Coding criteria in the taxonomy and inter-rater reliability

\begin{tabular}{|c|c|c|}
\hline \multirow[t]{4}{*}{$\begin{array}{l}\text { Performance expectancy } \\
\text { (IRR: 0.79) }\end{array}$} & High & $\begin{array}{l}\text { (Researchers conducted interview(s) before designing the CDS tool } \\
\text { OR Researchers performed usability testing with at least five users and more than } 75 \% \text { of participants agreed } \\
\text { the CDS tool was useful } \\
\text { OR Clinicians agreed that CDS tool provided patient-specific recommendation.) } \\
\text { AND The CDS tool was context sensitive (e.g., CDS triggered by workflow events and CDS targeted to the right } \\
\text { providers) }\end{array}$ \\
\hline & Medium & $\begin{array}{l}\text { (Researchers performed usability testing with at least five users and 50\%-75\% of participants agreed the CDS } \\
\text { tool was useful } \\
\text { OR The CDS tool was context sensitive.) } \\
\text { AND Local users were involved in developing the CDS tool [33] }\end{array}$ \\
\hline & Low & $\begin{array}{l}\text { Researchers performed usability testing and less than } 50 \% \text { participants agreed the CDS tool was useful } \\
\text { OR Local users were involved in developing the CDS tool [33] } \\
\text { OR The CDS tool was context sensitive }\end{array}$ \\
\hline & Unknown & Not enough relevant information mentioned in the article \\
\hline \multirow[t]{4}{*}{$\begin{array}{l}\text { Effort expectancy } \\
\text { (IRR: 0.85) }\end{array}$} & High & $\begin{array}{l}\text { The CDS tool interrupted the workflow } \\
\text { OR The CDS tool required a lot of effort to use (e.g., providing the dashboard, requesting documentation of } \\
\text { reason for any non-compliance [33], and at least some manual input of values [34]) } \\
\text { OR The CDS tool required clinician initiative to use [33] } \\
\text { OR The estimated user interaction time was greater than } 3 \mathrm{~s} \\
\text { OR } 75 \%+\text { of users reported that the CDS tool required high effort to use }\end{array}$ \\
\hline & Medium & $\begin{array}{l}\text { The CDS tool interrupted the workflow and required moderate cognitive load (e.g., users needed to click one or } \\
\text { few buttons to accept or dismiss, had a pop-up window to display the message) } \\
\text { OR The estimated user interaction time was } 1-3 \mathrm{~s} \\
\text { OR } 50-74 \% \text { of users reported that the CDS tool required high effort to use }\end{array}$ \\
\hline & Low & $\begin{array}{l}\text { The CDS tool was quick and required less than } 1 \mathrm{~s} \text { to use. Users did not need to find information somewhere } \\
\text { else } \\
\text { OR } 50 \%+\text { of users reported that the CDS tool was easy to use }\end{array}$ \\
\hline & Unknown & Not enough relevant information mentioned in the article \\
\hline \multirow[t]{4}{*}{$\begin{array}{l}\text { Social influence } \\
\text { (IRR: 0.71) }\end{array}$} & High & $\begin{array}{l}\text { Peer pressure existed during the implementation (e.g., rated user's performance, patient was encouraged to be } \\
\text { involved in the decision) } \\
\text { OR Participants knew that they were being monitored on an individual basis (e.g., written justification was vis- } \\
\text { ible in the EHR [35], 1-to-1 feedback) } \\
\text { OR CDS was a part of a larger program implementation (e.g., falls program) } \\
\text { OR CDS was part of a regulatory measure (e.g., electronic clinical quality improvement) }\end{array}$ \\
\hline & Medium & $\begin{array}{l}\text { Researchers provided an education program for all of the users } \\
\text { OR Users received a communication from the supervisor about use of the CDS tool } \\
\text { OR CDS was targeted toward more than one provider of practice group }\end{array}$ \\
\hline & Low & $\begin{array}{l}\text { No tracking or no monitoring existed in the program } \\
\text { OR Researchers provided an education program for some users } \\
\text { OR Researchers did not provide an education program } \\
\text { OR CDS was targeted toward patients }\end{array}$ \\
\hline & Unknown & Not enough relevant information mentioned in the article \\
\hline Facilitating conditions & & $\begin{array}{l}\text { A. IT infrastructure or user customization (e.g., tailored the timing and frequency of prompts, tailored text, high- } \\
\text { lighted text, links to supporting information, make justification publicly visible, and interactive dashboard) } \\
\text { B. IT support for users (e.g., technical assistance to address hardware and software issues) } \\
\text { C. Training (e.g., the specialized instruction concerning the system provided) } \\
\text { D. Other facilitating factors (e.g., economics decrease the cost for the unnecessary tests) }\end{array}$ \\
\hline \multirow[t]{4}{*}{$\begin{array}{l}\text { Motivational control } \\
\text { (IRR: } 0.87 \text { ) }\end{array}$} & High & $\begin{array}{l}\text { Users could simply ignore the CDS tool, (e.g. low control on their behavior, passive alerts, and a changeable alert } \\
\text { threshold) }\end{array}$ \\
\hline & Medium & Users had to respond to the CDS tool but involved low effort, (e.g. pop up with easy exit out) \\
\hline & Low & $\begin{array}{l}\text { Users were forced to use the CDS tool with at least medium effort (e.g., pop up with text input, interrupt the } \\
\text { workflow, and cannot ignore it) }\end{array}$ \\
\hline & Unknown & Not enough relevant information mentioned in the article \\
\hline
\end{tabular}

IRR, inter-rater reliability; CDS, clinical decision support; IT, information technology

In this case, we explain why we classified the provision of laboratory data as having a low level of performance expectancy. While a CDS with lab-specific data might increase usefulness, in developing the taxonomy, we found that most of the current CDS research has recognized the importance of introducing more patientspecific data and have implemented such CDS in clinical practice. Therefore, the more important information 
Table 3 Coding of the first case-drug-drug interaction alert (Duke, 2013 [36])

\begin{tabular}{|c|c|c|c|c|}
\hline Performance expectancy & Effort expectancy & Social influence & Facilitating conditions & Motivational control \\
\hline \multicolumn{5}{|l|}{$\begin{array}{l}\text { Control: Drug-drug interaction } \\
\text { alert }\end{array}$} \\
\hline $\begin{array}{l}\text { Low } \\
\text { Trigger by drug-drug interac- } \\
\text { tions associated with } \\
\text { hyperkalemia }\end{array}$ & $\begin{array}{l}\text { Low } \\
\text { The interaction time less than } \\
\text { one second }\end{array}$ & $\begin{array}{l}\text { Low } \\
\text { No education or tracking } \\
\text { program }\end{array}$ & 1) provide explanation links & $\begin{array}{l}\text { Medium } \\
\text { Need user response; low } \\
\text { efforts }\end{array}$ \\
\hline \multicolumn{5}{|l|}{$\begin{array}{l}\text { Intervention: Drug-drug } \\
\text { interaction alert tailored with } \\
\text { specific patient laboratory } \\
\text { data (most recent potassium } \\
\text { and creatinine levels) }\end{array}$} \\
\hline $\begin{array}{l}\text { Low } \\
\text { Show relevant lab values with } \\
\text { the alert }\end{array}$ & $\begin{array}{l}\text { Low } \\
\text { The interaction time less than } \\
\text { one second }\end{array}$ & $\begin{array}{l}\text { Low } \\
\text { No education or tracking } \\
\text { program }\end{array}$ & $\begin{array}{l}\text { 1) provide explanation links; } \\
\text { 2) tailored alert text }\end{array}$ & $\begin{array}{l}\text { Medium } \\
\text { Need user response; low } \\
\text { efforts }\end{array}$ \\
\hline \multicolumn{5}{|c|}{ Change between study arms } \\
\hline- & - & - & +1 & - \\
\hline
\end{tabular}

for determining the level of performance expectancy is whether or not usability testing was conducted. It ensures that the additional information provided meets user needs and avoids information overload. It is also important to note that CDS should not simply list patientspecific information. Rather, the CDS should provide a recommendation based on the patient-specific information in order to be useful. In this study, the CDS would have been more useful from the clinician's perspective if it had provided specific recommendations based on the different values of the laboratory data, rather than just displaying values.

The second case shows a dynamic relationship between effort expectancy and motivational control (Table 4). Scheepers-Hoeks et al. compared CDS on demand with a pop-up alert for medications in the intensive care setting [37]. This comparison decreased effort expectancy from high to low and motivational control from high to medium. In the CDS-on-demand arm, effort expectancy was high, because providers needed to remember to activate the CDS in the EHR. With the pop-up alert, information was shown automatically, which decreased effort expectancy. Motivational control was high in the control arm, because providers could ignore the CDS tool. In the arm of pop-up alerts, the motivational control was medium, because the pop-up alert required users to respond with low effort. The CDS tools in both arms were locally customized and provided patient-specific recommendations, which supported high performance expectancy. Social influence was medium in both arms because of the educational program provided to all users, which would convey social or work obligations. After changing an on-demand CDS to a pop-up alert, providers could use the tool with less effort; however, they lost control to some degree. This case also shows the necessity of adding the motivational control in the model to characterize CDS interventions comprehensively. Changing a CDS on demand to a pop-up alert would decrease the effort to use the tool but also affect user autonomy and perceived control.

In the third case, Meeker et al. assessed the effect of three behavioral interventions to reduce inappropriate

Table 4 Coding of the second case-medication CDS in the ICU (Scheepers-Hoeks, 2013 [37])

\begin{tabular}{|c|c|c|c|c|}
\hline Performance expectancy & Effort expectancy & Social influence & Facilitating conditions & Motivational control \\
\hline \multicolumn{5}{|l|}{ Control: CDS on demand as an EHR section } \\
\hline $\begin{array}{l}\text { High } \\
\text { Customized, patient-specific recommendation }\end{array}$ & $\begin{array}{l}\text { High } \\
\text { Users have to remem- } \\
\text { ber to use the CDS }\end{array}$ & $\begin{array}{l}\text { Medium } \\
\text { Education program }\end{array}$ & $\begin{array}{l}\text { (1) education; } \\
\text { (2) tailored alert text }\end{array}$ & $\begin{array}{l}\text { High } \\
\text { Simply ignore }\end{array}$ \\
\hline \multicolumn{5}{|l|}{ Intervention: Pop-up alerts } \\
\hline $\begin{array}{l}\text { High } \\
\text { Customized, patient-specific recommendation } \\
\text { Change between study arms }\end{array}$ & $\begin{array}{l}\text { Low } \\
\text { Pop up automatically }\end{array}$ & $\begin{array}{l}\text { Medium } \\
\text { Education program }\end{array}$ & $\begin{array}{l}\text { (1) education; } \\
\text { (2) tailored alert text }\end{array}$ & $\begin{array}{l}\text { Medium } \\
\text { Pop-up alerts, easy exit out }\end{array}$ \\
\hline- & High $\rightarrow$ Low & - & - & High $\rightarrow$ Medium \\
\hline
\end{tabular}


antibiotic prescribing [35] (Table 5). Suggested alternatives alone were compared to the combination of suggested alternatives with accountable justification and peer comparison. Providers in the first arm received order sets to notify the user of suggested treatments. In the second arm, a prompt to enter a justification for overriding the alert was added to the suggested alternatives. Providers also received email feedback that provided their antibiotic prescribing rates and the lowest inappropriate prescribing rates from peers. This intervention changed effort expectancy and social influence from medium to high, motivational control from medium to low, and added two facilitating conditions. Effort expectancy was increased because of the need for manual input. Two aspects caused social influence to increase: one was the publicly visible justification, and another was peer pressure. Motivational control was decreased because providers were required to respond with high effort. The two facilitating conditions included feedback on inappropriate prescriptions and a requirement for public justification. This case emphasizes the importance to apply the UTAUT model with the motivational control to design or optimize CDS. In this study, researchers added two more behavioral intentions, however, the change between study arms didn't have an impact on the performance expectancy.

\section{Discussion}

We found that a taxonomy based on the UTAUT model and motivational control could not only be used to reliably characterize CDS interventions in the primary literature, but also to characterize the effect and an estimated mechanism of action. Moreover, adding the motivational control construct was needed to address the variation in how CDS interventions impact user autonomy. In validating the taxonomy, we achieved substantial to near perfect agreement when classifying CDS features in model constructs. The UTAUT model constructs and motivational control can be applied systematically and reliably to the CDS domain while providing generalizable knowledge. A future analysis of the larger CDS domain will allow us to evaluate the importance and predictive value of these theoretical constructs on outcomes. This work should encourage researchers to formally test specific hypotheses and theoretical mechanisms to understand the impact of CDS features.

Our modification of the UTAUT model, by adding motivation control, is congruent with other research in this area. While the UTAUT model was developed to robustly explain adoption and use of IT, researchers have been encouraged to modify the model based on unique characteristics of users and technology [9]. For instance, Alaiad and Zhou added a trust construct when developing a theoretical model of patient acceptance of a health care robot [38]. Hoque et al. added technology anxiety and resistance to change to examine factors that affect the adoption of mHealth by the elderly [39]. Another study adapted UTAUT by adding compatibility and self-efficacy constructs to explain acceptance and satisfaction of nurses using an EHR [40]. Finally, Chang et al. predicted providers' intention to use an online reporting system in 2012 by combining

Table 5 Coding of the third case-CDS to reduce inappropriate antibiotic prescribing (Meeker, 2016 [35])

\begin{tabular}{|c|c|c|c|c|}
\hline Performance Expectancy & Effort Expectancy & Social Influence & Facilitating Conditions & Motivational Control \\
\hline \multicolumn{5}{|c|}{$\begin{array}{l}\text { Control: Order sets to notify suggested } \\
\text { treatments }\end{array}$} \\
\hline $\begin{array}{l}\text { Low } \\
\text { Context sensitive }\end{array}$ & $\begin{array}{l}\text { Medium } \\
\text { Interrupt workflow, mod- } \\
\text { erate cognitive load }\end{array}$ & $\begin{array}{l}\text { Medium } \\
\text { Train all users }\end{array}$ & $\begin{array}{l}\text { (1) education; } \\
\text { (2) drop-down list; } \\
\text { (3) link to order sets; } \\
\text { (4) link to documentation tem- } \\
\text { plates }\end{array}$ & $\begin{array}{l}\text { Medium } \\
\text { Response with low efforts }\end{array}$ \\
\hline \multicolumn{5}{|c|}{$\begin{array}{l}\text { Intervention: Order sets to notify } \\
\text { suggested treatments, accountable } \\
\text { justification, and peer comparison }\end{array}$} \\
\hline $\begin{array}{l}\text { Low } \\
\text { Context sensitive }\end{array}$ & $\begin{array}{l}\text { High } \\
\text { Write, high cognitive load }\end{array}$ & $\begin{array}{l}\text { High } \\
\text { Public written } \\
\text { justification, peer } \\
\text { pressure }\end{array}$ & $\begin{array}{l}\text { (1) education; } \\
\text { (2) drop-down list; } \\
\text { (3) link to order sets; } \\
\text { (4) link to documentation tem- } \\
\text { plates; } \\
\text { (5) feedback on inappropriate } \\
\text { prescriptions; } \\
\text { (6) public justification }\end{array}$ & $\begin{array}{l}\text { Low } \\
\text { Pop-up with text input }\end{array}$ \\
\hline \multicolumn{5}{|l|}{ Change between study arms } \\
\hline- & Medium $\rightarrow$ High & Medium $\rightarrow$ High & +2 & Medium $\rightarrow$ Low \\
\hline
\end{tabular}


the UTAUT model with the value of perceived consequence, which can explain users' subjective values when using the system [10]. In the IT domain with the context of EHRs, modification of the UTAUT model was needed to account for the variable levels of autonomy offered by IT applications.

Our approach differs from other studies in four aspects. First, we operationalized the UTAUT constructs specifically for the CDS context using a formal approach of developing a reliable taxonomy. Second, we operationalized the constructs in terms of CDS features and not subjective judgments. This variation in how constructs are operationalized is not unusual in the experimental psychology literature, as most experimental studies directly test the casual impact of psychological mechanisms through explicit manipulations designed to create that effect. Of course, future work should both manipulate variables and measure their psychological impact subjectively and on behavior. Third, we applied the UTAUT model to investigate features from user psychological perspective. Comparing with the socio-technical system models (e.g. SEPIS [41]), our method targeted on individual behavior and their acceptance to a technology. Socio-technical system models often provide components and identify interactions between user, technology, and organization to better design or assess a whole system. Fourth, we selected RCTs in comparative effectiveness studies to develop and validate the taxonomy. This study type compares two CDS interventions with and without some features, which could provide more accurate evidence in evaluating the features' impacts on CDS success [42].

Limitations of this study should be noted. First, in many respects, the accuracy of coding results is influenced by the reporting quality of the selected RCTs. For example, if a study did not report conducting user interviews in the selected study or a previous study, then we assumed they did not conduct interviews. Second, our study used the CDS literature to develop the taxonomy instead of conducting surveys. Publication bias is likely prevalent in the CDS literature. Third, when researchers use our findings to guide CDS design and implementation, they need to be cautious and carefully examine the overall usefulness of the system carefully. For example, when evaluating performance expectancy, we had two clinicians to determine whether the CDS tool provided patient-specific recommendations. The pre-implementation testing (e.g. usability testing) should not be ignored. Finally, constructs in the UTAUT model and motivational control have not been empirically validated in their ability to predict adoption behavior and patient outcomes. Our next step is to conduct meta-regression to explore how constructs relate to the effectiveness of CDS interventions.

\section{Conclusion}

This study demonstrated the feasibility of characterizing CDS interventions in terms of a behavioral theory. We successfully developed a reliable coding schema using a validated behavioral theory model for HIT. The provided taxonomy can be used to create generalizable knowledge from the primary literature and guide future CDS development, implementation, and evaluation to maximize the chance of user acceptance and CDS adoption.

\begin{abstract}
Abbreviations
CDS: Clinical decision support; HIT: Health information technology; IT: Information technology; UTAUT: Unified Theory of Acceptance and Use of Technology; k: Kappa; EHR: Electronic health record; RCTs: Randomized controlled trials; IRR: Inter-rater reliability; AHCPR: Agency for Healthcare Policy and Research; CFIR: Consolidated Framework for Implementation Research; PHARIS: Promoting Action on Research Implementation in Health Services.
\end{abstract}

\section{Acknowledgements}

None.

\section{Authors' contributions}

Substantial contributions to the conception and study design: CW, KK, TR, SL, GDF. Substantial contributions to data acquisition and data analysis: SL, TR, CW. Substantial contributions to draft the manuscript: SL, TR, CW. Substantial contributions to revise the manuscript: CW, TR, GDF, KK, SL. All authors have approved the final manuscript.

Funding

This study was supported by the University of Utah.

\section{Availability of data and materials}

All data generated or analyzed during this study are included in this published article.

\section{Declarations}

Ethics approval and consent to participate Not applicable.

\section{Consent for publication}

Not applicable.

\section{Competing interests}

KK reports honoraria, consulting, sponsored research, licensing, or co-development outside the submitted work in the past three years with McKesson InterQual, Hitachi, Pfizer, Premier, Klesis Healthcare, RTI International, Mayo Clinic, Vanderbilt University, the University of Washington, the University of California at San Francisco, MD Aware, and the U.S. Office of the National Coordinator for Health IT (via ESAC and Security Risk Solutions) in the area of health information technology. KK was also an unpaid board member of the non-profit Health Level Seven International health IT standard development organization, he is an unpaid member of the U.S. Health Information Technology Advisory Committee, and he has helped develop a number of health IT tools which may be commercialized to enable wider impact. None of these relationships have direct relevance to the manuscript but are reported in the interest of full disclosure.

Received: 30 October 2020 Accepted: 2 March 2021

Published online: 17 March 2021

References

1. Moja L, Kwag KH, Lytras T, Bertizzolo L, Brandt L, Pecoraro V, et al. Effectiveness of computerized decision support systems linked to electronic 
health records: a systematic review and meta-analysis. Am J Public Health. 2014;104:e12-22.

2. Kawamoto K, Houlihan CA, Balas EA, Lobach DF. Improving clinical practice using clinical decision support systems: a systematic review of trials to identify features critical to success. BMJ. 2005;330:765.

3. Osheroff JA, Teich JM, Middleton B, Steen EB, Wright A, Detmer DE. A roadmap for national action on clinical decision support. J Am Med Inform Assoc. 2007;14:141-5.

4. Roshanov PS, Fernandes N, Wilczynski JM, Hemens BJ, You JJ, Handler $S M$, et al. Features of effective computerised clinical decision support systems: meta-regression of 162 randomised trials. BMJ. 2013;346:1-12.

5. Van de Velde S, Kunnamo I, Roshanov P, Kortteisto T, Aertgeerts B, Vandvik PO, et al. The GUIDES checklist: development of a tool to improve the successful use of guideline-based computerised clinical decision support. Implement Sci. 2018;13:86.

6. Van de Velde S, Heselmans A, Delvaux N, Brandt L, Marco-Ruiz L, Spitaels $D$, et al. A systematic review of trials evaluating success factors of interventions with computerised clinical decision support. Implement Sci. 2018;13:114.

7. Bright TJ, Wong A, Dhurjati R, Bristow E, Bastian L, Coeytaux RR, et al. Effect of clinical decision-support systems. Ann Intern Med. 2012;157:29.

8. Shekelle PG, Morton SC, Keeler EB. Costs and benefits of health information technology. Evid Rep Technol Assess (Full Rep). 2006;132:1-71.

9. Venkatesh V, Morris MG, Davis GB, Davis FD. User acceptance of information technology: toward a unified view. MIS Q. 2003;27:425.

10. Chang $\mathrm{I}-\mathrm{C}$, Hsu H-M. Predicting medical staff intention to use an online reporting system with modified unified theory of acceptance and use of technology. Telemed e-Health. 2012;18:67-73.

11. Brighton B, Bhandari M, Tornetta P, Felson DT. Hierarchy of evidence: from case reports to randomized controlled trials. Clin Orthop Relat Res. 2003:413:19-24.

12. Bailey KD. Typologies and taxonomies: an introduction to classification techniques. London: Sage Publications; 1994.

13. Oh J-C, Yoon S-J. Predicting the use of online information services based on a modified UTAUT model. Behav Inf Technol. 2014;33:716-29.

14. Cimino JJ. Improving the electronic health record_-Are clinicians getting what they wished for? JAMA. 2013;309:991.

15. Deci EL, Ryan RM. The 'what' and 'why' of goal pursuits: human needs and the self-determination of behavior. Psychol Inq. 2000;11:227-68.

16. Bandura A. Human agency in social cognitive theory. Am Psychol. 1989;44:1175-84.

17. Csikszentmihalyi M. Beyond boredom and anxiety. Jossey-Bass; 2000.

18. Emaeilzadeh P, Sambasivan M, Nezakati H. The limitations of using the existing TAM in adoption of clinical decision support system in hospitals. Int J Res Bus Soc Sci. 2014;3:56-68.

19. Walter Z, Lopez MS. Physician acceptance of information technologies: role of perceived threat to professional autonomy. Decis Support Syst. 2008;46:206-15.

20. Esmaeilzadeh P. Interaction with clinical decision support systems: the challenge of having a steak with no knife. In: eHealth and remote monitoring. InTech; 2012.

21. Esmaeilzadeh P, Sambasivan M, Kumar N, Nezakati H. Adoption of clinical decision support systems in a developing country: antecedents and outcomes of physician's threat to perceived professional autonomy. Int J Med Inform. 2015;84:548-60.

22. Sambasivan M, Esmaeilzadeh P, Kumar N, Nezakati H. Intention to adopt clinical decision support systems in a developing country: effect of Physician's perceived professional autonomy, involvement and belief: a cross-sectional study. BMC Med Inform Decis Mak. 2012;12:142.

23. Ajzen I. From intentions to actions: a theory of planned behavior. In: Action control. Springer, Berlin; 1985. p. 11-39.

24. Hill RJ, Fishbein M, Ajzen I. Belief, attitude, intention, and behavior: an introduction to theory and research. Contemp Sociol. 1977;6:244.
25. Fillmore CL, Rommel CA, Welch BM, Zhang M, Kawamoto K, Lake S, et al. The perils of meta-regression to identify clinical decision support system success factors. J Biomed Inform. 2016;56:65-8.

26. Lobach D, Sanders GD, Bright TJ, Wong A, Dhurjati R, Bristow E, et al. Enabling health care decisionmaking through clinical decision support and knowledge management. Evid Rep Technol Assess (Full Rep). 2012;1-784.

27. Garg AX, Adhikari NKJ, McDonald H, Rosas-Arellano MP, Devereaux PJ, Beyene J, et al. Effects of computerized clinical decision support systems on practitioner performance and patient outcomes: a systematic review. JAMA. 2005;293:1223-38.

28. Chang I-C, Hwang H-G, Hung W-F, Li Y-C. Physicians' acceptance of pharmacokinetics-based clinical decision support systems. Expert Syst Appl. 2007;33:296-303.

29. Damschroder LJ, Aron DC, Keith RE, Kirsh SR, Alexander JA, Lowery JC. Fostering implementation of health services research findings into practice: a consolidated framework for advancing implementation science. Implement Sci. 2009;4:50.

30. Kitson A, Harvey G, McCormack B. Enabling the implementation of evidence based practice: a conceptual framework. Qual Saf Heal Care. 1998;7:149-58.

31. Cohen J. A coefficient of agreement for nominal scales. Educ Psychol Meas. 1960;20:37-46.

32. McHugh ML. Interrater reliability: the kappa statistic. Biochem Medica. 2012:22:276-82.

33. Kawamoto K, Lobach DF. Clinical decision support provided within physician order entry systems: a systematic review of features effective for changing clinician behavior. In: AMIA Annual Symposium proceedings AMIA Symposium. 2003;361-5.

34. Miller K, Capan M, Weldon D, Noaiseh Y, Kowalski R, Kraft R, et al. The design of decisions: matching clinical decision support recommendations to Nielsen's design heuristics. Int J Med Inform. 2018;117:19-25.

35. Meeker D, Linder JA, Fox CR, Friedberg MW, Persell SD, Goldstein NJ, et al. Effect of behavioral interventions on inappropriate antibiotic prescribing among primary care practices a randomized clinical trial. JAMA. 2016:315:562.

36. Duke JD, Li X, Dexter P. Adherence to drug-drug interaction alerts in highrisk patients: a trial of context-enhanced alerting. J Am Med Informatics Assoc. 2013:20:494-8.

37. Scheepers-Hoeks AMJ, Grouls RJ, Neef C, Ackerman EW, Korsten EH. Physicians' responses to clinical decision support on an intensive care unit-comparison of four different alerting methods. Artif Intell Med. 2013;59:33-8.

38. Alaiad A, Zhou L. Patients' behavioral intention toward using healthcare robots. Proc Ninet Am Conf Inf Syst. 2013;15-17:1-11.

39. Hoque R, Sorwar G. Understanding factors influencing the adoption of mHealth by the elderly: an extension of the UTAUT model. Int J Med Inform. 2017;2017(101):75-84.

40. Maillet É, Mathieu L, Sicotte C. Modeling factors explaining the acceptance, actual use and satisfaction of nurses using an Electronic Patient Record in acute care settings: an extension of the UTAUT. Int J Med Inform. 2015;84:36-47.

41. Carayon P, Schoofs Hundt A, Karsh B-T, Gurses AP, Alvarado CJ, Smith M, et al. Work system design for patient safety: the SEIPS model. Qual Heal Care. 2006;15(suppl 1):i50-8.

42. Armstrong K. Methods in comparative effectiveness research. J Clin Oncol. 2012;30:4208-14.

\section{Publisher's Note}

Springer Nature remains neutral with regard to jurisdictional claims in published maps and institutional affiliations. 